\title{
Human papillomavirus testing as an optional screening tool in low-resource settings of Latin America: experience from the Latin American Screening study
}

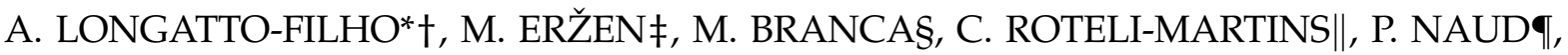 \\ S.F.M. DERCHAIN\#, L. HAMMESף, L.O. SARIAN\#, J.F. BRAGANÇA\#, J. MATOSฯ, \\ R. GONTIJO\#, T. LIMA\|, M.Y.S. MAEDA*, S. TATTI**, S. SYRJÄNEN\#, G. DORESłキ, \\ A. LÖRINCZ§§ \& K. SYRJÄNEN\# \\ *Pathology Division of Adolfo Lutz Institute, São Paulo, Brazil and Health Sciences Research Institute (ICVS), \\ School of Health Sciences, University of Minho, Braga, Portugal; tLife and Health Sciences Research Institute \\ (ICVS), School of Health Sciences, University of Minho, Braga, Portugal; ¥SIZE Diagnostic Center, Ljubljana, \\ Slovenia; §Istituto Superiore di Sanità, Rome, Italy; //Cervical Pathology Services, Hospital Leonor Mendes de \\ Barros, São Paulo, Brazil; 9 Department of Gynecology \& Obstetrics, Hospital de Clinicas de Porto Alegre, \\ Brazil; \#Centro de Atenção Integral à Saúde Da Mulher (CAISM), Universidade Estadual de Campinas, Brazil; \\ **Department of Gynecology \& Obstetrics, Hospital de Clinicas de Porto Alegre, Brazil; \#キDigene Brasil, \\ São Paulo, Brazil; §§Digen Corporation, Gaithersburg, Maryland
}

\begin{abstract}
Longatto-Filho A, Eržen M, Branca M, Roteli-Martins C, Naud P, Derchain SFM, Hammes L, Sarian LO, Bragança JF, Matos J, Gontijo R, Lima T, Maeda MYS, Tatti S, Syrjänen S, Dores G, Lörincz A, Syrjänen K. Human papillomavirus testing as an optional screening tool in low-resource settings of Latin America: experience from the Latin American Screening Study. Int J Gynecol Cancer 2006;16:955-962.

Hybrid capture II (HC II) test for oncogenic human papillomaviruses (HPV) was carried out in a cohort of 4284 women at their first clinical visit. Overall prevalence of HPV was $17.1 \%$, decreasing with age from $33.9 \%$ among women below 20 years to only $11.0 \%$ among those older than 41 years. HPV prevalence was significantly higher among current smokers (odds ratio $[O R]=1.31 ; 95 \%$ CI 1.1-1.6), in women with two or more lifetime sexual partners $(O R=1.9 ; 95 \%$ CI 1.6-2.4), and those women with two or more sexual partners during the past 12 months prior to examination (OR $=1.6 ; 95 \%$ CI 1.2-2.2). HPV detection increased in parallel with increasing cytologic abnormality, being highest in women with high-grade squamous intraepithelial lesion $(P=0.001)$. Specificity of the HPV test in detecting histologically confirmed cervical disease was 85\% (95\% CI 83.9-86.1). Sensitivity of the HPV test in detecting histologic abnormalities increased in parallel with disease severity, ranging from $51.5 \%$ for cervical intraepithelial neoplasia (CIN) 1 to $96.5 \%$ for CIN 3 and $100.0 \%$ for cancer, with respective decline of positive predictive value. These data suggest that HPV testing with HC II assay might be a viable screening tool among this population with relatively high prevalence of cervical disease.
\end{abstract}

KEYWORDS: cervical cancer, HPV testing, low-resource setting, screening.

Address correspondence and reprint requests to: Adhemar LongattoFilho, MSc, PhD, PMIAC, Life and Health Sciences Research Institute, School of Health Sciences, University of Minho, Campus de Gualtar, 4710-057 Braga, Portugal. Email: longatto16@hotmail.com
In the recent literature, a variety of new strategies for cervical cancer screening are heavily debated. There is no doubt that the organized screening based on cervical cytology has resulted in dramatic reduction of incidence and mortality of cervical cancer in countries where implemented ${ }^{(1,2)}$, despite the recognized limitations of this diagnostic tool, ie, false-negative 
smears ${ }^{(3)}$. According to the usual practice, women with high-grade squamous intraepithelial lesion (HSIL) are referred for colposcopy ${ }^{(4)}$, while repeated smear is recommended in cases with mild cytologic abnormalities atipy of squamous cells (ASC) or low-grade squamous intraepithelial lesion (LSIL). Due to the fact that a) ASC and LSIL are common findings (with only $10 \%$ representing true high-grade lesions) ${ }^{(5)}$, b) falsepositive tests result in substantial number of unnecessary colposcopies ${ }^{(4,6)}$, and c) false-negative smears and failure to detect high-grade lesions and even cancer, this strategy is responsible for a heavy burden to the public health care system.

Presence of high-risk human papillomaviruses (HPV) is necessary for the development and persistence of cervical intraepithelial neoplasia $(\mathrm{CIN})^{(7,8)}$. This has prompted extensive testing of different HPV detection assays as optional screening tools of cervical cancer. Thus, addition of HPV testing to cytology has been suggested to increase the sensitivity and negative predictive values (NPV) for high-grade cervical lesions, therefore, prolonging the screening intervals and reducing referrals for colposcopy ${ }^{(9-12)}$, making HPV testing also a cost-effective option ${ }^{(13)}$. Based on these favorable recent data, national guidelines, eg, in United States now allow the combined use of HPV testing and cytology in the primary screening of women 30 years or older ${ }^{(14,15)}$. In several European countries, the incorporation of HPV testing in cervical cancer screening is currently under discussion ${ }^{(16,17)}$.

Incidence of cervical cancer in Latin America is among the highest worldwide ${ }^{(18)}$. However, disease burden in this large continent is unevenly distributed, being dependent upon the screening practices in the different regions. These range from practical nonavailability to relatively well-organized programs, most notably in the urban centers of the south of the continent. The authors recently designed a multicenter study to test eight different screening tools in a cohort of over 12,000 women enrolled by four clinics in regions of Brazil and Argentina with different incidence of cervical cancer, known as the Latin American Screening (LAMS) study ${ }^{(19)}$. One of the two major aims of this study is to find out the cost-effective tools for cervical cancer screening in these low-resource settings.

HPV testing using commercially available hybrid capture II (HC II) is one of the eight tools included in the LAMS study ${ }^{(19)}$. The present communication reports our experience with the use of HC II assay in over 4000 Brazilian and Argentinean women and critically weights the possible utility of this assay in cervical cancer screening, in regions with relatively high incidence of cervical disease.

\section{Materials and methods}

\section{Study design}

LAMS study is an ongoing prospective multicenter study, supported by the European Commission through its International Cooperation for Development Countries (INCO-DEV) Program (ICA4-CT2001$10013)^{(19)}$. In this study, 12,114 consecutive women from the cities of Campinas (Brazil), São Paulo (Brazil), Porto Alegre (Brazil), and Buenos Aires (Argentina) were recruited to undergo gynecological examination and testing with conventional Papanicolaou (Pap) smear, visual inspection with acetic acid (VIA), visual inspection with Lugoĺs iodine (VILI), cervicography, and screening colposcopy. Women were sampled for HPV testing by HC II. To ensure high reproducibility of the testing, specialist gynecologists carried out examinations in all centers and well-trained nurses took care of the specimen collection for HC II and Pap. All enrolled women gave their agreement to participate by signing the informed consent, written in their native language. The study protocol has been approved by the local ethics committees of all four participating clinics.

Women were considered eligible if they met all of the following requirements: a) had an intact uterus (ie, no previous surgical procedure of the cervix or corpus); b) had no history of abnormal Pap test in the past year; c) were not under treatment for genital condyloma (external or in the cervix); d) had no sexual intercourse during the 3 days prior to the examination; and e) did not have any confirmed or clinically suspect immunosuppression (human immunodeficiency virus, corticosteroids, chemotherapy) or other chronic diseases that might compromise the immune system.

Flowchart of the patient examination is shown in Figure 1. At the first visit, all women were subjected to a questionnaire addressing clinical and epidemiologic risk factors of cervical neoplasia. All women were subjected to a thorough pelvic examination, comprising collection of the Pap smear, HC II sampling, and VIA or VILI (data not shown). Women who had at least one of these examinations abnormal were referred to colposcopic examination. Also, to control for verification bias, $5 \%$ of the women with all screening tests negative were randomly assigned for colposcopy and all (802 women) who attended consultations at Campinas State University Hospital were subjected to colposcopy. Whenever colposcopy revealed an abnormal pattern, a directed punch biopsy was taken ${ }^{(19)}$. Women had their second visit scheduled after 45 days, to become informed about their exam/biopsy results and to be allotted to either the treatment or the follow-up 


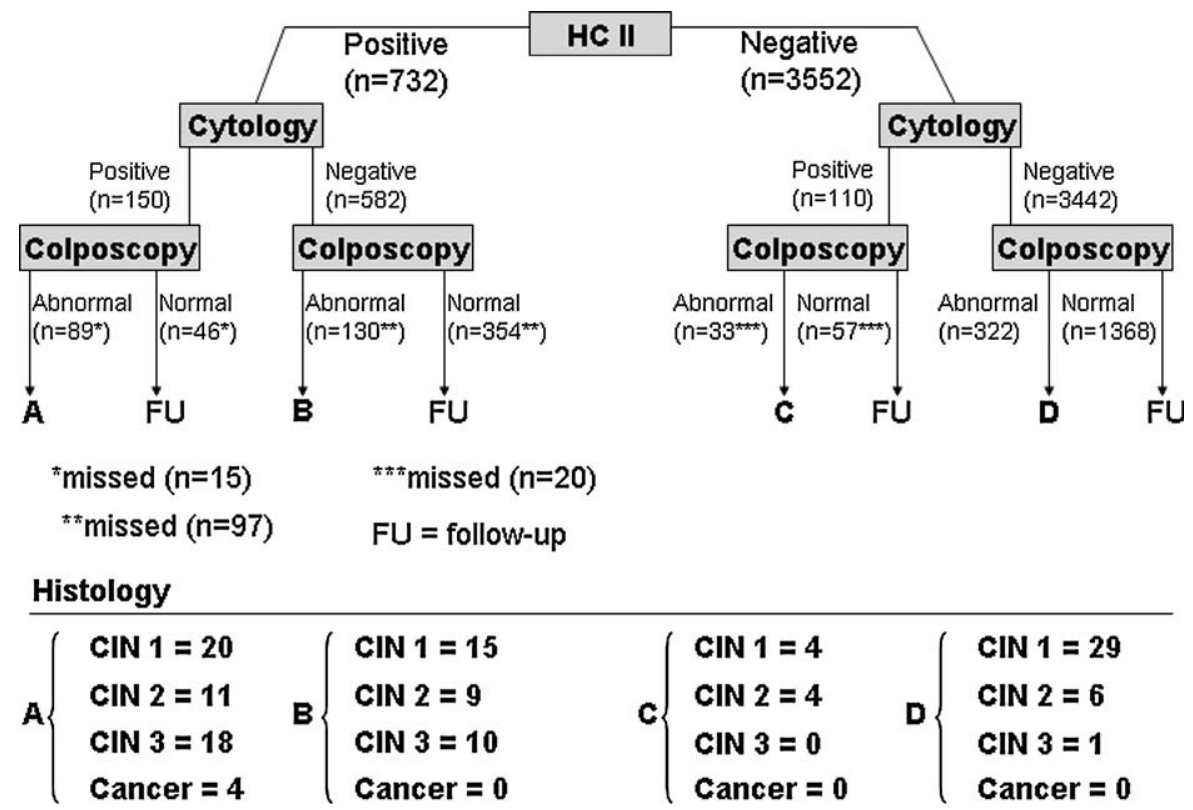

Figure 1. Flowchart of the examinations and their results.

group. Treatment was offered in accordance to the routine practices adopted by the participating centers.

\section{Study centers and demographics}

Campinas (Brazil) is a 1 million inhabitants city, situated in southeast region of the country, approximately $100 \mathrm{~km}$ from São Paulo city. The city is a dynamic commercial and industrial center, with a relatively well-structured health system and some high-standard hospitals. However, a substantial portion (almost 20\%) of its population, living in the outskirts of the city, is composed of people who migrated from the north and northeast (the poorest) regions of the country, searching for jobs. In São Paulo state, encompassing the cities of Campinas and São Paulo, cervical cancer is the fourth major cause of cancer death among women, accounting for $3.3 \%$ of all female deaths due to cancer. In this region, breast cancer accounted for $13.3 \%$ of cancer deaths among women between 1995 and $1999^{(15)}$. Women have been enrolled in the Centro de Atenção Integral à Saúde da Mulher, a State University of Campinas' teaching hospital, dedicated to women care and in a basic health unit in the outskirts of the city. São Paulo city is the economic powerhouse of Brazil, with 11 million inhabitants. Its population is composed of a multiple ethnic groups (European whites, Asians, and Afro-Americans). Health care is heterogeneous, ranging from overcrowded public basic health units and hospitals to a high-quality private sector. In this city, women have been enrolled by
Hospital Leonor Mendes de Barros, a public institution that performs over 50,000 gynecological and obstetric consultations every year. Porto Alegre (Brazil) is the capital of Rio Grande do Sul State, in the south of the country. The state's population enjoys the best levels of quality of life in the country although cervical cancer is the sixth major cause of cancer death among women, accounting for $6.10 \%$ of all female deaths due to cancer. In this region, breast cancer accounted for $15.1 \%$ of cancer deaths among women from 1995 to $1999^{(15)}$. The participant center was Hospital de Clínicas de Porto Alegre, a general hospital affiliated to Federal University of Rio Grande do Sul. The Department of Obstetrics and Gynecology of this center is responsible for roughly 30,000 gynecological and obstetric consultations per year. Buenos Aires is the capital of Argentina, and the participating center in this country was Hospital de Clínicas José de San Martin, a general teaching hospital with large experience in gynecology and obstetrics and being a reference center for colposcopy for the entire country. The country has an overall cervical cancer mortality rate of 7.6 per 100,000 women $^{(16)}$.

\section{Examinations}

\section{Cervical cytology (Pap smear)}

Conventional Pap smears were taken using the Ayre spatula and endocervical brush, fixed in 95\% ethanol, and stained by the modified Pap method. For liquid-based 
cytology, a direct-to-vial protocol was followed (DNACitoliq System, Digene, São Paulo, Brazil; and AutocytePREP, TriPath Imaging, Burlington, NC). Final cytologic diagnoses were issued by the Bethesda 2001 System $^{(20)}$ and were classified as normal/inflammatory, ASC, atypical glandular cells, LSIL, or HSIL.

\section{Hybrid capture II (HC II)}

Altogether, 4284 women were tested with HC II at their first clinical visit. The specimens for HC II were tested with probe B (high-risk HPVs: types 16, 18, 31, $33,35,39,45,51,52,56,58,59$, and 68$)^{(21)}$, and the tests were classified positive at the relative light unit/ positive control (RLU/CO) ratio of $1 \mathrm{pg} / \mathrm{mL}$ or greater. These $\mathrm{RLU} / \mathrm{CO}$ ratios provide a semiquantitative estimate of the amount of HPV DNA in the specimens, ie, the viral load in the sample. Storage of specimens and reagents, as well as test processing, was carried out in manufacturer-certified laboratories, under the responsibility of the investigators, following the manufacturer's instructions (Digene Co. Gaithersburg, MD). São Paulo (Brazil) and Buenos Aires (Argentina) processed their own HC II samples inhouse, while Campinas (Brazil) and Porto Alegre (Brazil) had their HC II specimens processed at a Campinas University Hospital laboratory.

\section{Cervical biopsies}

Directed punch biopsies (and cone biopsies) were fixed in formalin, embedded in paraffin, and processed into $5-\mu \mathrm{m}$-thick sections stained with hematoxylin and eosin for light microscopy, following the routine procedures. All biopsies were examined among the daily routine in the Pathology Departments of the four clinics and diagnosed using the commonly agreed CIN nomenclature. For the study purposes, the pathologists were also asked to notify the morphologic changes suggestive for the presence of HPV in cases with no CIN (flat condyloma).

\section{Statistical analysis}

Possible differences regarding patients' age distribution between study centers were tested with the paired $t$ test. After testing age distribution for normality with Shapiro-Wilk's test (normality has been ruled-out), age differences across the strata were assessed with nonparametric tests (Wilcoxon's rank sum test). Logistic regression analysis was used to calculate odds ratio (OR) for HC II positivity related to the recorded epidemiologic variables, as well as to cytologic (Pap smear) or histologic results. The $95 \%$ confidence intervals (95\% CI) were calculated for all data using the accurate methods ${ }^{(22)}$.

\section{Results}

Mean age of the patients was 37.5 years (90\% central range 20.4-55.4 years). Overall prevalence of HPV was $17.1 \%$. Mean age of women with positive HC II (33.6 years) was significantly lower than that of women with a negative HPV test (38.3 years; $P<0.0001$ ). HPV prevalence decreased with age, ranging from $33.9 \%$ among women younger than 20 years to only $11.0 \%$ among those aged 41 years or more, with no difference between the studied four populations $(P=$ 0.90; Table 1).

HPV detection related to key epidemiologic characteristics of the patients is shown in Table 2. Current smoking was marginally related with HPV infection $(\mathrm{OR}=1.31 ; 95 \%$ CI 1.1-1.6). As compared to women who had only one lifetime sexual partner, women with two to four lifetime sexual partners $(\mathrm{OR}=1.9$; 95\% CI 1.6-2.4) and those who had five or more sexual partners $(\mathrm{OR}=2.7 ; 95 \% \mathrm{CI} 2.1-3.5)$ presented with

Table 1. Age-related detection ${ }^{a}$ of HPV in the four clinics

\begin{tabular}{lccrrr}
\hline & \multicolumn{1}{l}{ Clinic $^{b}$} & & & \\
\cline { 2 - 6 } Age (years) & $\begin{array}{l}\text { São Paulo, } \\
\text { Positive/total (\%) }\end{array}$ & $\begin{array}{l}\text { Campinas, } \\
\text { Positive/total (\%) }\end{array}$ & $\begin{array}{l}\text { Porto Alegre, } \\
\text { Positive/total (\%) }\end{array}$ & $\begin{array}{l}\text { Buenos Aires, } \\
\text { Positive/total (\%) }\end{array}$ & $\begin{array}{l}\text { Total, } \\
\text { Positive/total (\%) }\end{array}$ \\
\hline$\leq 20$ & $2 / 18(11.0)$ & $42 / 127(33.1)$ & $14 / 25(56.0)$ & $3 / 10(30.0)$ & $61 / 180(33.9)$ \\
$21-30$ & $50 / 187(26.7)$ & $115 / 463(24.8)$ & $43 / 156(27.6)$ & $56 / 264(21.2)$ & $264 / 1070(24.7)$ \\
$31-40$ & $38 / 253(15.0)$ & $64 / 389(16.5)$ & $50 / 304(16.4)$ & $53 / 258(20.5)$ & $205 / 1204(17.0)$ \\
$\geq 41$ & $43 / 348(12.4)$ & $49 / 457(10.7)$ & $63 / 618(10.2)$ & $47 / 408(11.5)$ & $202 / 1830(11.0)$ \\
Total $^{c}$ & $133 / 806(16.5)$ & $270 / 1436(18.8)$ & $170 / 1103(15.4)$ & $159 / 940(16.9)$ & $732 / 4284(17.1)$ \\
\hline
\end{tabular}

${ }^{a} \mathrm{HC} \mathrm{II}>=1.0 \mathrm{RLU} / \mathrm{CO}$.

${ }^{b}$ Comparing study centers: $P=0.90$ (excluding patients 20 years old or younger).

${ }^{c}$ Mean age of women with positive HC II (33.6 years) was significantly lower than that of women with a negative HPV test (38.3 years, $P<0.0001)$. 
Table 2. HPV detection related to key characteristics of the patients

\begin{tabular}{|c|c|c|c|}
\hline \multirow[b]{2}{*}{ Characteristic } & \multicolumn{2}{|l|}{ HC II } & \multirow[b]{2}{*}{$\begin{array}{l}\text { Adjusted }^{a} \\
\text { OR(95\% CI) }\end{array}$} \\
\hline & $\begin{array}{l}\text { Positive, } \\
n(\%)\end{array}$ & $\begin{array}{l}\text { Negative, } \\
n(\%)\end{array}$ & \\
\hline \multicolumn{4}{|l|}{ Age (years) } \\
\hline$\leq 20$ & $61(8.3)$ & $119(3.4)$ & $4.4(3.1-6.5)$ \\
\hline $21-30$ & $264(36.1)$ & $806(22.7)$ & $2.3(1.8-2.9)$ \\
\hline $31-40$ & $205(28.0)$ & $999(28.1)$ & $1.5(1.2-1.9)$ \\
\hline$\geq 41$ & $202(27.6)$ & $1628(45.8)$ & Referential \\
\hline \multicolumn{4}{|c|}{ Current smoker } \\
\hline Yes & $206(28.1)$ & 765 (21.5) & $1.3(1.1-1.6)$ \\
\hline No & $526(71.9)$ & $2787(78.5)$ & Referential \\
\hline \multicolumn{4}{|c|}{ Oral contraceptives } \\
\hline Yes & $271(37.1)$ & $1100(31)$ & $1.2(0.9-1.4)$ \\
\hline No & 459 (62.9) & $2452(69)$ & Referential \\
\hline \multicolumn{4}{|l|}{ Parity } \\
\hline 0 & $339(46.3)$ & 1396 (39.3) & Referential \\
\hline $1-2$ & $263(35.9)$ & $1368(38.5)$ & $1.0(0.8-1.2)$ \\
\hline 3 or more & $130(17.8)$ & $792(22.3)$ & $1.1(0.9-1.5)$ \\
\hline \multicolumn{4}{|c|}{ Currently pregnant } \\
\hline Yes & $20(2.7)$ & $58(1.6)$ & $1.2(0.7-2.0)$ \\
\hline $\mathrm{No}$ & $692(94.5)$ & 3437 (96.6) & Referential \\
\hline \multicolumn{4}{|l|}{$\begin{array}{l}\text { Lifetime sexual } \\
\text { partners }\end{array}$} \\
\hline 1 & $181(24.7)$ & $1555(43.7)$ & Referential \\
\hline $2-4$ & $385(52.6)$ & $1553(43.7)$ & $1.9(1.6-2.4)$ \\
\hline$\geq 5$ & $166(22.7)$ & 449 (12.6) & $2.7(2.1-3.5)$ \\
\hline \multicolumn{4}{|l|}{$\begin{array}{l}\text { Sexual partners } \\
\text { during past } \\
12 \text { months }\end{array}$} \\
\hline 0 or 1 & $656(89.6)$ & $3422(96.2)$ & Referential \\
\hline$\geq 2$ & $76(10.4)$ & $136(3.8)$ & $1.6(1.2-2.2)$ \\
\hline
\end{tabular}

${ }^{a}$ Logistic regression.

a significantly higher HPV prevalence. HPV was also more prevalent among women with two or more sexual partners during the past 12 months prior to consultation, as compared with women who had no or only one sexual partner (OR $=1.6$; $95 \%$ CI 1.2-2.2).

Table 3. HPV detection as related to Pap smear abnormalities

\begin{tabular}{|c|c|c|c|c|}
\hline \multirow[b]{2}{*}{ Pap smear } & \multicolumn{2}{|l|}{ HC II } & \multirow[b]{2}{*}{ OR } & \multirow[b]{2}{*}{$95 \% \mathrm{CI}$} \\
\hline & $\begin{array}{l}\text { Positive, } \\
n(\%)\end{array}$ & $\begin{array}{l}\text { Negative, } \\
n(\%)\end{array}$ & & \\
\hline Normal & $582(14.5)$ & 3442 (85.5) & Ref & \\
\hline ASC & 65 (41.6) & 91 (58.4) & 2.9 & $2.4-3.5$ \\
\hline LSIL & $43(78.2)$ & $12(21.8)$ & 21.4 & $10.8-43.1$ \\
\hline HSIL & 38 (92.7) & $3(7.3)$ & 75.6 & $22.3-307.2$ \\
\hline AGC & $3(50.0)$ & $3(50.0)$ & 6.0 & $0.9-37.0$ \\
\hline $\begin{array}{l}\text { Invasive } \\
\text { carcinomas* }\end{array}$ & $1(50.0)$ & $1(50.0)$ & $\mathrm{n} / \mathrm{a}$ & $\mathrm{n} / \mathrm{a}$ \\
\hline Total & 732 (17.1) & $3552(82.9)$ & - & - \\
\hline
\end{tabular}

AGC, atypical glandular cells.

${ }^{*} \mathrm{P}<0.001$.

$\mathrm{n} / \mathrm{a}=$ not available.
Parity, being pregnant at the time of testing, and current use of oral contraceptives did not show any relation to HPV positivity.

Table 3 shows HPV detection rates in women with different Pap smear abnormalities. ORs increased in parallel with progressive cytologic abnormalities: ASC $(\mathrm{OR}=2.9 ; 95 \%$ CI $2.4-3.5)$, LSIL $(\mathrm{OR}=21.4 ; 95 \% \mathrm{CI}$ 10.8-43.1), and HSIL (OR = 75.6; 95\% CI 22.3-307.2). Only six women were diagnosed with glandular abnormalities in cytology, which were not statistically associated with increased HPV detection ( $\mathrm{OR}=6.0$; 95\% CI 0.9-37.0).

HPV detection is related to histologic diagnosis (Table 4). Fifteen percent of women with no detectable disease had a positive HC II test, making the test specific at the level of $85 \%$ (95\% CI 83.9\%-86.1\%). HPV prevalence increased in parallel with increasing grade of the lesions, ending up with $100 \%$ in invasive carcinomas $(P<0.001)$. Sensitivity of the HPV test to histologic abnormalities increased in parallel with disease severity, ranging from $51.5 \%$ for CIN 1 to $96.5 \%$ for CIN 3 and $100.0 \%$ for cancer.

\section{Discussion}

Recent developments in understanding the natural history of cervical cancer and its precursors as well as the rapid development of novel technologies are challenging the traditional concepts of cervical cancer screening. Until now, most studies addressing HPV detection as a screening tool have been carried out in developed countries, where prevalence of cervical abnormalities has been modified by several years of well-conducted screening programs using cervical cytology ${ }^{(23-25,13)}$. The ongoing LAMS study is one of the first multicenter efforts conducted in developing countries ${ }^{(19)}$, thus, providing important insights into the utility of HC II testing in populations in lowresource settings.

In alignment with most of the previous reports, HC II assay is highly sensitive for high-grade cervical disease. Of 29 women with CIN 3, only 1 was HPV negative and all the 4 cases of cancer had a positive HPV test. The likelihood of a woman with negative HPV testing to harbor a high-grade cervical lesion is extremely low, and thus, the NPV of HPV testing is expected to be very high ${ }^{(24,26)}$. Solid confirmation of these findings has been provided by the Population Based Screening Study Amsterdam (POBASCAM) in which 44,102 women were tested for high-risk HPV types using polymerase chain reaction (PCR $)^{(25)}$. In the intervention group (women subjected to HPV testing plus cytology) of this population-based controlled 
Table 4. HPV detection as related to histologically confirmed cervical pathology

\begin{tabular}{|c|c|c|c|c|c|c|}
\hline \multirow[b]{2}{*}{ Final diagnosis } & \multicolumn{2}{|l|}{ HPV DNA } & \multirow[b]{2}{*}{ Sensitivity $(95 \% \mathrm{CI})$} & \multirow[b]{2}{*}{ Specificity $(95 \%$ CI) } & \multirow[b]{2}{*}{ PPV (\%) } & \multirow[b]{2}{*}{ NPV (\%) } \\
\hline & Positive, $n(\%)$ & Negative, $n(\%)$ & & & & \\
\hline No disease & $644(15.0)$ & $3508(85.0)$ & - & $85 \%(83.9-86.1)$ & - & 97.1 \\
\hline HPV /CIN 1 & $35(51.5)$ & $33(48.5)$ & $51.5 \%(39.6-63.3)$ & & 5.4 & - \\
\hline CIN 2 & $21(67.7)$ & $10(33.3)$ & $67.7 \%(61.3-84.2)$ & & 3.3 & - \\
\hline CIN 3 & $28(96.5)$ & $1(3.5)$ & $96.5 \%(89.9-100.0)$ & & 4.3 & - \\
\hline Invasive carcinomas* & $4(100.0)$ & $0(0.0)$ & $100.0 \%(100.0-100.0)$ & & 0.7 & - \\
\hline Total & $732(100.0)$ & $3552(100.0)$ & - & & - & - \\
\hline
\end{tabular}

${ }^{*} P<0.001$.

trial, $96.7 \%$ (89 out of 92 cases) of women diagnosed with CIN 3 or worse lesions had a positive HPV test. The comparisons of LAMS study data and POBASCAM study, however, should be taken with caution because POBASCAM is a population-based trial, and the women invited for LAMS cohort study should be free of HPV-induced disease at least for 1 year. This conceptual difference between both populations can explain, in part, the overall HPV prevalences: 5\% in POBASCAM study, contrasting to the $17.1 \%$ of the present series. We can hypothesize if this marked difference in HPV prevalence portrays any substantial differences in the risk of cervical cancer between the populations studied by POBASCAM ${ }^{(25)}$ and in the LAMS study, the Dutch and Latin American women, respectively. These risk discrepancies, clearly favoring the Dutch population, is a mere reflection of the epidemiologic factors involved with HPV infection. Mean age at first intercourse, number of sexual partners, and parity, directly addressed in this study, are known to be, by data reported on several population series, dramatically disadvantageous to Latin American populations as compared to Western European ones.

Another interesting feature in comparing the LAMS and the POBASCAM studies was the marked similarity in the prevalence of cytologic abnormalities as related to the HPV status. In the LAMS cohort, 79.5\% (582/732) of the women with positive HPV test showed a normal cytology, the corresponding figure in the POBASCAM study being $69.4 \%{ }^{(25)}$. Accordingly, borderline and mild dyskaryosis were found in $16.8 \%$ of the HPV-positive POBASCAM population, being almost identical with that (14.9\%) of ASC/LSIL in the present series. However, $13.7 \%$ of the HPV-positive POBASCAM women had HSIL, as contrasted to only $5.2 \%$ in the present series. Ultimately, the prevalence of histologically confirmed cervical disease in the LAMS study is almost fivefold higher than that in the POBASCAM study ${ }^{(25)}$. This cannot be due to the different performance of HC II and PCR used in the
LAMS and POBASCAM study, respectively, because there seem to be no differences. It should be noted that the HPV positivity for CIN 3 lesions in the POBASCAM study was $96.7 \%$ and in this study $96.5 \%$. It is noteworthy that no glandular lesions (ie, in situ or invasive adenocarcinoma) were found in the present series, even though there were six cases of glandular abnormalities in Pap smears. Although the viral types included in both tests are almost identical (only HPV 66 is detected by HC II but not by the PCR), PCR is usually more analytically sensitive than HC II; however, the clinical sensitivity of HC II is usually at least equal to that of $\mathrm{PCR}^{(25)}$. Therefore, for the women of LAMS cohort had not being properly triaged before and for the socioeconomic unfavorable circumstances in which these women live, high prevalence of HPV might had led to an augmented prevalence of actual HPV-related disease. It is possible to speculate that immunologic factors, related to impoverished living conditions, smoking habits, and inadequate triaging and treatment of vaginal infections, probably favored the development of disease in HPV-infected women.

The adoption of any new screening tool and practice should be matched with the local cost environment, expertise, and existing facilities. This fact has been well recognized recently and has prompted researchers to design and implement studies aimed at testing different optional screening tools that are affordable in the local settings of their implementation. For instance, most studies on visual inspection of the cervix (VIA or VILI) have been conducted in India and Africa, where cervical disease is highly prevalent and limitations of resources are dramatic ${ }^{(27,28)}$. The regions of Brazil and Argentina, where this study is being carried out do not equal in disease burden with the Indian and African settings used to test these two visual methods. However, they do have higher prevalence of cervical cancer than most European and North American countries, where most of the studies on HPV testing have been conducted ${ }^{(27,28)}$. This fact probably makes the present results applicable for all the settings, facing 
a transition between an underdeveloped and a developed world. Indeed, such settings can be encountered in several regions of South America and Asia.

Currently, cost-effectiveness is one of the major concerns of health authorities planning public health programs. In countries with high living standards, such as the UK, the Netherlands, France, and Italy, screening with HPV testing has been proven cost-effective in a recently published study ${ }^{(13)}$. Those researchers concluded that HPV DNA testing has the potential to improve health benefits at a reasonable cost in the aforementioned countries, with an overall incremental cost of $\$ 13,000$ per year of life saved. This incremental cost is believed unacceptable for a majority of the developing countries, especially with regard to governmentfunded programs.

Unfortunately, to compare the costs of the current Brazilian public health system reimbursement for Pap smear with HC II is not possible because the Brazilian government does not reimburse HC II yet. Currently, the reimbursement for Pap smear for hospitals and laboratories is R $\$ 5.37$, which is approximately US $\$ 2.0$ for each cervical Pap smear performed. The cost of HC II in Brazil is approximately U $\$ 7-\mathrm{U} \$ 8$ for public sector screening projects. Nowadays, the use of HC II in Latin America is strictly tied to the private health assurance sector that is free to stipulate the value to be reimbursed. Indeed, this fact limits the use of HC II, as recently discussed ${ }^{(19)}$.

Regardless of these cost restrictions, high prevalence of HPV in the present cohort results in the extremely low positive predictive value (PPV) of the HC II test. PPV along with NPV is believed to be crucial parameters to screening of any disease. It is widely regarded that the observed PPV should be a reflection of the tests performance characteristics in providing an acceptable false-positive rate and an NPV with robust potential to avoid an unacceptable false-negative rate. In other words, test performance characteristics in terms of PPV and NPV need to meet an acceptable compromise $^{(29)}$. This is true for Latin America where we have a high prevalence of histologically confirmed disease. If we consider that in the past three decades, the rates of morbidity and mortality for cervical cancer did not change in many regions of Brazil for several reasons, sensitivity and NPV should be taken into account as important parameter to prevent disease and to select women for specific tools to recognize disease. Actually, to overcome this major shortcoming of the HC II assay and to take the full advantage of the high sensitivity of the test, the most reasonable approach is to combine cytology and HPV testing. Indeed, combined use of cytology and HPV testing is the rationale of the current recommendations for the HPV test usage in the United States ${ }^{(14)}$. A negative HPV result together with normal cytology renders the women eligible for the next screening round after 3 years or more, resulting in a considerable reduction in the number of medical consultations. Conversely, a positive HPV test alone shall not mandate a colposcopic examination in populations with high prevalence of $\mathrm{HPV}^{(4,14)}$, such as the LAMS study cohort.

As suggested in several studies, women aged 30 years or more would be the appropriate targets of HPV-based screening strategies ${ }^{(4,14)}$. This also applies to the LAMS study cohort if HPV testing is coupled to a reflex cytology test. Roughly, $14 \%$ of women in the higher age groups were HPV positive in this study, which therefore precludes any possible usage of HPV testing in this population as a stand-alone screening tool, with immediate referral of positive women to colposcopy because of the obvious overload of colposcopy referrals inevitably resulting from such a policy.

Current research on cervical screening is being focused on innovative use of traditional and novel diagnostic tools ${ }^{(24,27,28)}$. For the moment, there does not seem to be any stand-alone test capable of delivering optimal sensitivity combined with good predictive values. Therefore, combined use of available diagnostic tools shall be considered, while planning novel screening strategies. In this planning, the designers need to be aware of the weaknesses and strengths of each individual test, as related to their performance in the target populations to be screened. This study provides some insights into the performance of HPV testing (with an Food and Drug Administration (USA)-approved commercially available HC II assay) in a population with a relatively high prevalence of HPV-related cervical disease. Screening strategies optimally tailored for different public health environments critically depend on testing of several optional diagnostic tools under field conditions, as done in the ongoing LAMS study.

\section{Acknowledgments}

This study has been supported by the European Commission, INCO-DEV Program (Contract number ICA4-CT-2001-10013). The generous contribution of Digene Co. (USA), donating the HC II tests at our disposal, is gratefully acknowledged.

\section{References}

1 Bergström R, Sparen P, Adami HO. Trends in cancer of the cervix uteri in Sweden following cytological screening. $\mathrm{Br} J$ Cancer 1999;81:159-66. 
2 Vizcaino AP, Moreno V, Bosch FX et al. International trends in incidence of cervical cancer: II. Squamous-cell carcinoma. Int J Cancer 2000;86:429-35.

3 Nanda K, McGregory DC, Myers ER et al. Accuracy of the Papanicolaou test in screening for and follow-up of cervical cytologic abnormalities: a systematic review. Ann Intern Med 2000;132:810-9.

4 Wright TC Jr, Cox JT, Massad LS, Twiggs LB, Wilkinson EJ; ASCCPSponsored Consensus Conference. 2001 Consensus Guidelines for the management of women with cervical cytological abnormalities. JAMA 2002;287:2120-9.

5 Schiffman M, Solomon D. Findings to date from the ASCUS-LSIL Triage Study (ALTS). Arch Pathol Lab Med 2003;127:946-9.

6 Apgar BS, Zoschnick L, Wright TC Jr. The 2001 Bethesda System terminology. Am Fam Physician 2003;68:1992-8.

7 International Agency for Research on Cancer (IARC) Working group. Human papillomaviruses. Vol. 64. Lyon, France: World Health Organization, IARC, 1995.

8 Walboomers JM, Jacobs MV, Manos MM et al. Human papillomavirus is a necessary cause of invasive cervical cancer worldwide. J Pathol 1999;189:12-9.

9 Kulasingam SL, Hughes JP, Kiviat NB et al. Evaluation of human papillomavirus testing in primary screening for cervical abnormalities: comparison of sensitivity, specificity, and frequency of referral. JAMA 2002;288:1749-57.

10 Sherman ME, Lorincz AT, Scott DR et al. Baseline cytology, human papillomavirus testing, and risk for cervical neoplasia: a 10-year cohort analysis. J Natl Cancer Inst 2003;95:46-52.

11 Petry KU, Menton S, Menton M et al. Inclusion of HPV testing in routine cervical cancer screening for women above 29 years in Germany: results for 8466 patients. Br J Cancer 2003;88:1570-7.

12 Lorincz AT, Richart RM. Human papillomavirus DNA testing as an adjunct to cytology in cervical screening programs. Arch Pathol Lab Med 2003;127:959-68.

13 Kim JJ, Wright TC, Goldie SJ. Cost-effectiveness of human papillomavirus DNA testing in the United Kingdom, The Netherlands, France, and Italy. J Natl Cancer Inst 2005;97:888-95.

14 Wright TC Jr, Schiffman M, Solomon D et al. Interim guidance for the use of human papillomavirus DNA testing as an adjunct to cervical cytology for screening. Obstet Gynecol 2004;103:304-9.

15 Ministério da Saúde-Instituto Nacional do Câncer. Atlas de Mortalidade por Câncer no Brasil 1979-1999. Rio de Janeiro. INCA, 2002. Available at: http://www.inca.gov.br/atlas/docs/Atlas_completo.pdf [only Portuguese].

16 Argentina, Ministry of Health. Estadísticas vitales. Información básica año 2000. Buenos Aires, Argentina: Dirección de Estadísticas e formación de Salud, 2000.
17 Van den Akker-van Marle ME, van Ballegooijen M, van Oortmarssen GJ, Boer R, Habbema JD. Cost-effectiveness of cervical cancer screening: comparison of screening policies. I Natl Cancer Inst 2002;94:193-204.

18 International Agency for Research on Cancer. Cancer incidence in five continents. Vol. 5. Lyon, France: IARC Press, 2002.

19 Syrjänen K, Naud P, Derchain SM et al. Comparing PAP smear cytology, aided visual inspection, screening colposcopy, cervicography and HPV testing as optional screening tools in Latin America. Study design and baseline data of the LAMS study. Anticancer Res 2005;25:3469-80.

20 Solomon D, Davey D, Kurman R et al. The 2001 Bethesda System. Terminology for reporting results of cervical cytology. JAMA 2002;287:2114-9.

21 Lörincz AT, Castle PE, Sherman ME et al. Viral load of human papillomavirus and risk of CIN 3 or cervical cancer. Lancet 2002; 360:288-9.

22 R Development Core Team. $R$ : A language and environment for statistical computing. ISBN 3-900051-00-3. Vienna, Austria: R Foundation for Statistical Computing, 2004. Available at: http://www.R-project.org.

23 Cox JT, Lorincz AT, Schiffman $\mathrm{MH}$ et al. Human papillomavirus testing by hybrid capture appears to be useful in triaging women with cytologic diagnosis of atypical squamous cells of undetermined significance. Am J Obstet Gynecol 1995;172:946-54.

24 Clavel C, Masure M, Bory JP et al. Human papillomavirus testing in primary screening for the detection of high-grade cervical lesions: a study of 7932 women. Br J Cancer 2001;84:1616-23.

25 Bulkmans NW, Rozendaal L, Snidjers PJ et al. POBASCAM, a population-based randomized controlled trial for implementation of high-risk HPV testing in cervical screening: design, methods and baseline data of 44,102 women. Int J Cancer 2004;110:94-101.

26 Manos MM, Kinney WK, Hurley LB et al. Identifying women with cervical neoplasia: using human papillomavirus DNA testing for equivocal Papanicolaou results. JAMA 1999;281:1605-10.

27 Denny L, Kuhn L, Pollack A et al. Direct visual inspection for cervical cancer screening: an analysis of factors influencing test performance. Cancer 2002;94:1699-707.

28 Sankaranarayanan R, Nene BM, Dinshaw K et al. Early detection of cervical cancer with visual inspection methods: a summary of completed and on-going studies in India. Salud Publica Mex 2003;45(Suppl. 3):S399-407.

29 dos Santos Silva I. Cancer epidemiology: principles and methods. Lyon, France: IARC, 1999: 355-80.

Accepted for publication November 15, 2005 